\title{
Prevalence of vaginolysin, sialidase and phospholipase genes in Gardnerella vaginalis isolates between bacterial vaginosis and healthy individuals
}

\author{
Rokhsareh Mohammadzadeh ${ }^{1,2}$, Behrooz Sadeghi Kalani ${ }^{1,2}$, Maryam Kashanian $^{3}$, Mojgan Oshaghi $^{4}$, \\ Nour Amirmozafari*1,2
}

Received: 12 Nov 2018

Published: 19 Aug 2019

\section{Abstract}

Background: Gardnerella vaginalis is considered as the predominant microorganism found in bacterial vaginosis (BV). The aim of this study was to evaluate the prevalence of virulence factors in G. vaginalis associated with BV or non-BV cases and their correlations with this disorder.

Methods: A total of 102 vaginal specimens were collected from patients during their visit to Akbar Abadi hospital in Tehran, Iran. Bacterial vaginosis was determined by Nugent score and Amsel's criteria. Polymerase chain reaction (PCR) was used for the detection of $G$. vaginalis $16 \mathrm{~S}$ rRNA, vaginolysin, sialidase and phospholipase genes. To evaluate the association between the presence of $v l y$, pho, and sld genes and BV. Pearson Chi-square test was applied using SPSS software. P-value $\leq 0.05$ was considered as significant.

Results: Totally, $27.4 \%$ of the patients were suffering from BV. Gardnerella vaginalis was found in $100 \%$ women with BV and in $56.7 \%$ women with normal vaginal discharge. The prevalence of $v l y$, sld and pho genes in BV-associated G. vaginalis was 10 (35.7\%) $(95 \%$ CI $[0.18,0.53]), 19(67.8 \%)(95 \%$ CI $[0.51,0.85])$ and $6(21.4 \%)(95 \%$ CI $[0.06,0.37])$, respectively. The prevalence of the aforementioned genes in non-BV associated G. vaginalis was $20(47.6 \%)(95 \% \mathrm{CI}[0.33,0.63]), 28(66.6 \%)(95 \% \mathrm{CI}[0.52,0.81])$, and $5(11.9 \%)(95 \%$ CI $[0.02,0.22])$, respectively. Our results showed no statistically significant association between the presence of the virulence genes and $\mathrm{BV}$ associatedness of this microorganism.

Conclusion: Our results showed the presence of G. vaginalis in all BV patients and relatively high prevalence in healthy individuals. The prevalence rates of the three virulence genes were different in BV and non-BV associated G. vaginalis; however, the differences were not statistically significant.

Keywords: Gardnerella vaginalis, Bacterial vaginosis, PCR

Conflicts of Interest: None declared

Funding: Iran University of Medical Sciences

\section{*This work has been published under CC BY-NC-SA 1.0 license.}

Copyright $\odot$ Iran University of Medical Sciences

Cite this article as: Mohammadzadeh R, Sadeghi Kalani B, Kashanian M, Oshaghi M, Amirmozafari N. Prevalence of vaginolysin, sialidase and phospholipase genes in Gardnerella vaginalis isolates between bacterial vaginosis and healthy individuals. Med J Islam Repub Iran. 2019 (19 Aug);33:85. https://doi.org/10.47176/mjiri.33.85

\section{Introduction}

One of the most common problems among women of

Corresponding author: Dr Nour Amirmozafari, amirmozafari@iums.ac.ir

1. Microbial Biotechnology Research Center, Iran University of Medical Sciences, Tehran, Iran

2. Department of Microbiology, School of Medicine, Iran University of Medical Sciences, Tehran, Iran

3. Department of Obstetrics and Gynecology, School of Medicine, Iran University of Medical Sciences, Tehran, Iran

4. Department of Laboratory Sciences, School of Allied Health, Iran University of Medical Sciences, Tehran, Iran childbearing age leading to irregular vaginal discharge is

$\uparrow$ What is “already known" in this topic:

Gardnerella vaginalis is prevalently found in bacterial vaginosis causing several outcomes including infertility, preterm birth, and low birth. The pathogenicity of this microorganism has been attributed to several virulence factors.

\section{$\rightarrow$ What this article adds:}

There is no significant difference between the presence of virulence genes including vaginolysin, sialidase, and phospholipase in $G$. vaginalis isolates and the presence of bacterial vaginosis disorder; however, the expression levels may differ in healthy and bacterial vaginosis cases. Moreover, molecular identification of this microorganism was found to be more efficient compared with culture method. 
considered to be bacterial vaginosis (BV) (1). Several serious outcomes, including preterm parturition, infertility, and low birth weight have been reported to be linked with BV (2). This disorder may be a risk factor for predisposal of endometritis and sexually transmitted diseases, including HIV (3). Thin greyish-white vaginal discharge with a strong foul odor, and irritation in vulva and vagina are the most reported symptoms of BV (4).

Bacterial growth and the variety of bacteria are restricted by the presence of Lactobacilli which are considered as the major constituents of healthy vaginal microbiome, specifically those which are known to produce hydrogen peroxide, lactic acid and other toxic metabolites (5). In cases which the frequency of Lactobacillus is found to be low, the vaginal $\mathrm{pH}$ increases (6), leading to the overgrowth of microbes which are commonly found in BV, including Gardnerella vaginalis, Bacteroides spp, Mobiluncus spp, Mycoplasma hominis, Atopobium, Leptotrichia, Megaspaera, Prevotella, and Dialister $(7,8)$.

The predominant microorganism of the vaginal tract in women with BV is $G$. vaginalis (2) which was termed after Hermann L. Gardner, who discovered the bacterium in 1955. Gardnerella is in the family Bifidobacteriaceae and is mostly related to species in the genus Bifidobacterium (9). G. vaginalis has been isolated from the vaginal discharge of almost $100 \%$ of women with BV (10). Recent studies suggest that $G$. vaginalis may be more virulent than other organisms frequently found in this infection (4). Moreover, the existence of distinct pathogenic and non-pathogenic strains or even subspecies is proposed due to genetic differences of this bacterium (11). This organism may cause infections in sites other than genital tract of women, including urinary tract infections in men, infective endocarditis, septic emboli (12), retinal vasculitis (13), acute hip arthritis in renal transplant recipients (14), vertebral osteomyelitis, discitis (15) and bacteremia in a previously healthy man (16). Hence, it is believed to be an infrequent opportunistic pathogen owing to its various virulence factors.

One of these virulence factors is vaginolysin (VLY), a secreted protein toxin functioning as a hemolysin specific to human erythrocytes, neutrophils and endothelial cells (17). Similar to intermedilysin (ILY) secreted by Streptococcus intermedius (18) and lectinolysin derived from Streptococcus mitis (19), VLY is a cholesteroldependent pore-forming cytolysin (CDC) which recognizes the complement regulatory molecule CD59 on the surface of human cells. The VLY-CD59 interaction involves in the pathogenesis of $\mathrm{BV}$ and the consequent outcomes (20). Due to its ability of binding to complement proteins $\mathrm{C} 8 \alpha$ and $\mathrm{C} 9, \mathrm{CD} 59$ inhibits the formation of complement membrane attack complex (MAC). Membrane-bound monomers are oligomerized through conformational changes in domain 3 of the toxin occurred by hCD59-VLY binding; however, the cytolytic activity of the toxin still depends on the membrane cholesterol The cytolytic activity of vaginolysin strictly depends on cholesterol and is potentiated by human CD59 (21). Interestingly, cell lysis has still been observed in cells lacking hCD59 by VLY-cholestrol interaction accounting for the formation of oligomeric complex (22). Mutation of a proline residue which has been suggested to be necessary for the cytotoxicity of VLY potentiates the production of a VLY toxoid which may be used for further development of vaccines (17).

Another virulence gene in G. vaginalis is considered to be sialidase (or neuraminidase) that enzymatically removes terminal sialic acid residues from different glycoconjugates which provides bacteria with nutrition and improves their ability for evasion of the host immune system and cellular interactions (11). Pregnancy problems such as premature birth in BV patients have been attributed to sialidase activity owing to its mucin oligosaccharides degradation activity (23).

Phospholipase C (lecithinase or phosphatidylcholine phosphorylase) enzymatically hydrolyzes lecithin into phosphorylcholine and 1,2-diglyceride and is another recognized virulence factor of this microorganism. Lecithin is converted to 1, 2-diglyceride by phospholipase $\mathrm{C}$ activity. Arachidonic acid is further released through sequential actions which involve 1, 2 diglyceride and can consequently lead to the production of prostaglandins, thromboxanes, leukotrienes, and related compounds. The function of these oxygen metabolites affects childbirth, embryo implantation, coagulation, and inflammation. Phospholipase C-induced loss of structural integrity of cells. Accordingly, bacterial production of this enzyme can lead to reproductive tract cell and tissue damage through direct and indirect mechanisms (24).

The objective of this study was to look at the occurrence status of $G$. vaginalis and its virulence genes including vaginolysin ( $v l y)$, sialidase (sld) and phospholipase $\mathrm{C}$ (pho) in BV and non-BV cases. Evaluation of the virulence factors could be helpful for development of effective treatments.

\section{Methods}

Study population and sample collection: Participants were recruited among 102 non-pregnant women aged 1948 years seeking care at the gynecologic care unit of Akbar Abadi Hospital, Tehran, Iran, between June 2017 and January 2017. Exclusion criteria for this study included menstruation, menopause, and use of oral or vaginal antibiotics and topical vaginal products in the past 2 weeks. Normal and abnormal vaginal fluids were collected from the lateral vaginal sidewall after inserting a sterile, non-lubricated speculum. Informed written consent was obtained from each subject for agreement to participate in the study.

Determination of bacterial vaginosis: One of the swabs was rolled onto a glass slide for Gram stain evaluation. After the removal of the speculum, vaginal fluid remnants were used for the evaluation of $\mathrm{pH}$ and release of amine odor by the addition of $10 \%$ potassium hydroxide ("whiff test"). Bacterial vaginosis was defined clinically by the presence of homogeneous non-adherent grayish-white or yellow vaginal discharge, fishy odor in the presence of $10 \%$ potassium hydroxide, and vaginal $\mathrm{pH}>4.5$. The Gram-stained vaginal smears were examined under a 
light microscope at $100 \mathrm{X}$ in search of clue cells and lactobacilli. Gram variable or Gram negative coccobacilli were graded by a score developed by Nugent et al. (25). A score of $0-3$ is considered healthy (non-BV) and is characterized by the predominance of Gram-positive Lactobacillus morphotypes, a score of 4-6 is considered intermediate, and a score of $7+$ is indicative of $\mathrm{BV}$. Finally, BV is confirmed by the presence of distinctive clue cells in more than $20 \%$ of the total vaginal epithelial cells (26).

\section{Selective isolation of $G$. vaginalis}

One swab was placed in a test tube containing $1.0 \mathrm{~mL}$ phosphate-buffered saline (PBS) and another swab was immediately processed and rolled onto Columbia agar (Liofilchem, Italy) selective culture media supplemented with $0.01 \mathrm{mg} / \mathrm{L}$ nalidixic acid (Sigma-Aldrich $\mathrm{GmbH}$, Munich, Germany), $0.01 \mathrm{mg} / \mathrm{L}$ colistin (Sigma-Aldrich $\mathrm{GmbH}$, Munich, Germany), $0.004 \mathrm{mg} / \mathrm{L}$ amphotericin B (Sigma-Aldrich $\mathrm{GmbH}$, Munich, Germany) and human blood (5\%). Culture plates were then incubated under an anaerobic atmosphere, at $37^{\circ} \mathrm{C}$ for $48-72$ hours.

\section{Identification and confirmation of $G$. vaginalis}

Coupled with the presence of Gram-negative to Gramvariable rod bacteria under microscopy, biochemical tests including catalase, hippurate hydrolysis, starch hydrolysis, acid production from maltose and glucose, and alphaglucosidase activity were performed on small, white colonies with a beta-hemolytic zone for presumptive identification of $G$. vaginalis. Isolates were then confirmed by $16 S$ rRNA gene amplification and sequencing.

\section{DNA extraction}

Bacterial genomic DNA from culture and vaginal secretions (within $2 \mathrm{hr}$ ) were extracted using Genomic DNA Extraction Kit (JetFlex ${ }^{\mathrm{TM}}$, Löhne, Germany) according to the manufacturer's instructions. Evaluation of purity of the extracted DNA was carried out by a NanoDrop spectrophotometer (Thermo Fisher, USA). Genomic samples with an OD260/OD280 ratio of $\geq 1.8$ were applied for further analysis. Also, the quality of the extracted DNA was evaluated by $1 \%$ agarose gel electrophoresis (Sigma, USA).

Molecular identification of $G$. vaginalis and vaginolysin, phospholipase $C$ and sialidase genes

For specific molecular identification of $G$. vaginalis, polymerase chain reaction (PCR) was performed using specific $16 \mathrm{~S}$ rRNA primers. Specific primers were also designed and used for $v l y$, sld, and pho gene screening (Table 1). Amplification reactions were performed in an automated thermal cycler (Bio-Rad, USA) in a volume of $12.5 \mu \mathrm{l}$ consisting of $1 \mu \mathrm{l}$ of DNA template, $0.5 \mu \mathrm{l}$ of primers (forward and reverse), $6 \mu \mathrm{L}$ of Taq PCR Master Mix 2X (Fermentas, Lithuania) and $4.5 \mu \mathrm{L}$ of DNase/RNAse free distilled water (Thermo Fisher Scientific). The program consisted of an initial denaturation step at $95^{\circ} \mathrm{C}$ for 5 minutes, $30 \times 95^{\circ} \mathrm{C}$ for 45 sec; annealing (annealing Tm for each primer is shown in Table 1) for $50 \mathrm{sec}$; extension at $72^{\circ} \mathrm{C}$ for $45 \mathrm{sec}$ followed by a final extension at $72^{\circ} \mathrm{C}$ for 5 minutes. The amplicons in each reaction were analyzed on $1 \%$ agarose gel treated with safe stain (Greenview Plus, Andy Gold ${ }^{\mathrm{TM}}$, USA) in $0.5 \mathrm{X}$ TBE after electrophoresis. The gels were visualized under transilluminator UV light (Bio-Rad, UK).

Random sequencing of amplicons was performed for quality control and as a negative control, PCR reactions were performed without template DNA. The PCR products were sequenced (Macrogen, South Korea) after purification. The sequence data were analyzed using advanced BLAST search program at the NCBI database.

\section{Statistical Analysis}

In order to assess the association between the presence of vly, pho, and sld genes and BV-relatedness of G. vaginalis, Pearson Chi-square test was applied using SPSS version 24 . P-value $\leq 0.05$ was considered as significant.

\section{Results}

Isolation of $G$. vaginalis in vaginal samples: Among the 102 women, BV was diagnosed in 28 participants (prevalence of $27.45 \%$ ). The bacterium was cultured from 24 (19 BV and 5 non-BV) patients. Isolation of $G$. vaginalis by culture was not successful in all vaginal samples. Therefore, total DNA was extracted from vaginal secretions and used as PCR templates (Fig. 1). Amplification data showed the presence of $G$. vaginalis in vaginal secretions of 42 healthy individuals $(56.75 \%)$ and $28 \mathrm{BV}$ patients $(100 \%)$.

Detection of virulence genes: The prevalence of vly, sld and pho genes in BV-associated $G$. vaginalis was 10 $(35.7 \%)(95 \%$ CI $[0.18,0.53]), 19(67.8 \%)(95 \%$ CI [0.51, $0.85])$ and $6(21.4 \%)(95 \%$ CI [0.06, 0.37]), respectively. The prevalence of the aforementioned genes in non-BV associated G. vaginalis was $20(47.6 \%)(95 \%$ CI [0.33, $0.63]), 28(66.6 \%)(95 \%$ CI [0.52, 0.81]), and $5(11.9 \%)$ $(95 \%$ CI $[0.02,0.22])$, respectively (Figs. 2, 3, 4). Association between the presence of vly $(p=0.324)$, sld

Table 1. Characteristics of designed primers and related genes used in this study

\begin{tabular}{|c|c|c|c|c|}
\hline Targeted genes & Sequence $\left(5^{\prime} \longrightarrow 3^{\prime}\right)$ & $\begin{array}{l}\mathrm{Tm} \\
\left({ }^{\circ} \mathrm{C}\right)\end{array}$ & $\begin{array}{l}\text { Amplicon size } \\
\text { (bp) }\end{array}$ & Reference \\
\hline \multirow[t]{2}{*}{ 16S rRNA } & F GCTCAACCAGGCACAAAAACA & 59 & 300 & Present study \\
\hline & TCCACGCCTAGTTGGGTCTA & & & \\
\hline \multirow[t]{2}{*}{ Vaginolysin (vly) } & GCACCAGATAGCCCAGCAGA & 62 & 540 & Present study \\
\hline & R TTCGGTGCCGTACTCATCCC & & & \\
\hline \multirow[t]{2}{*}{ Phospholipase C (pho) } & F GCGTGCTCCGCTTCGATTAG & 61 & 421 & Present study \\
\hline & R TCCGCGGTAACGCTTCTCTT & & & \\
\hline \multirow[t]{2}{*}{ Sialidase (sld) } & F AGCCCGCATATCCCGTATCG & 62 & 454 & Present study \\
\hline & R GGACCTGGCCAACATGGAGT & & & \\
\hline
\end{tabular}




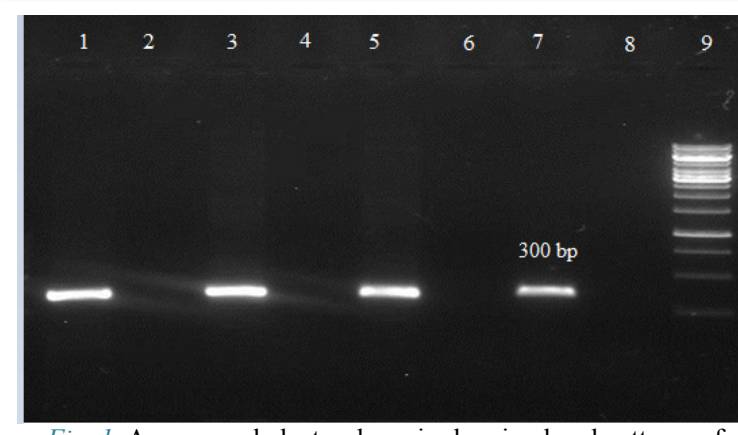

Fig. 1. Agarose gel electrophoresis showing band patterns of 16S rRNA gene for $G$. vaginalis

Lane 9: DNA marker (Ladder 1,000 bp)

Lane 7: positive control

Lane 8: negative control

Lanes 2, 4 and 6: DNA samples negative for 16S rRNA gene

Lanes 1, 3 and 5: DNA samples positive for 16S rRNA gene

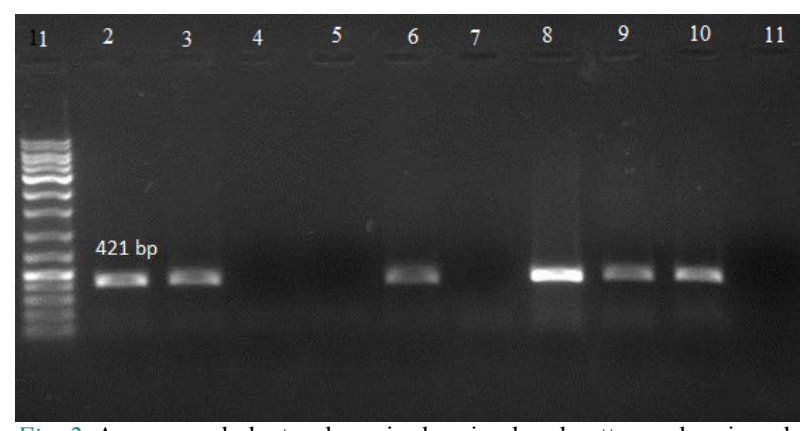

Fig. 2. Agarose gel electrophoresis showing band patterns showing pho gene Lane 1: DNA marker (Ladder $100 \mathrm{bp}$ )

Lane 10: positive control

Lane 11: negative control

Lanes 2, 3, 6, 8 and 9: DNA samples positive for pho gene

Lanes 4, 5 and 7: DNA samples negative for pho gene

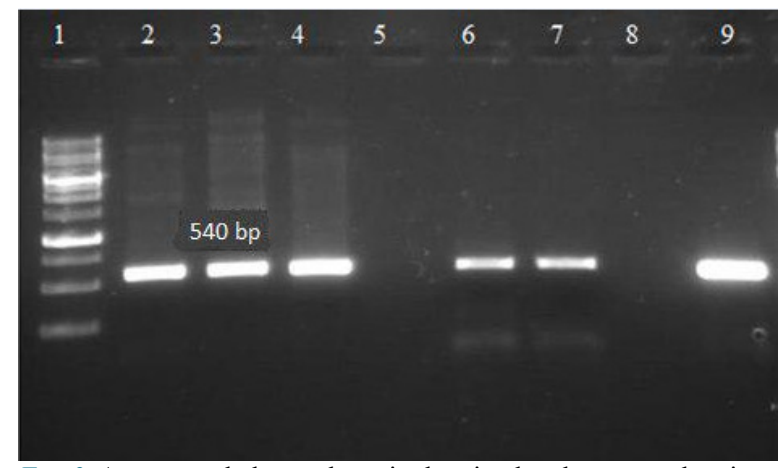

Fig. 3. Agarose gel electrophoresis showing band patterns showing vly gene

Lane 1: DNA marker (Ladder 1000bp)

Lane 8: negative control

Lane 9: positive control

Lanes 2-4, 6 and 7: DNA samples positive for vly gene

Lane 5: DNA samples negative for vly gene

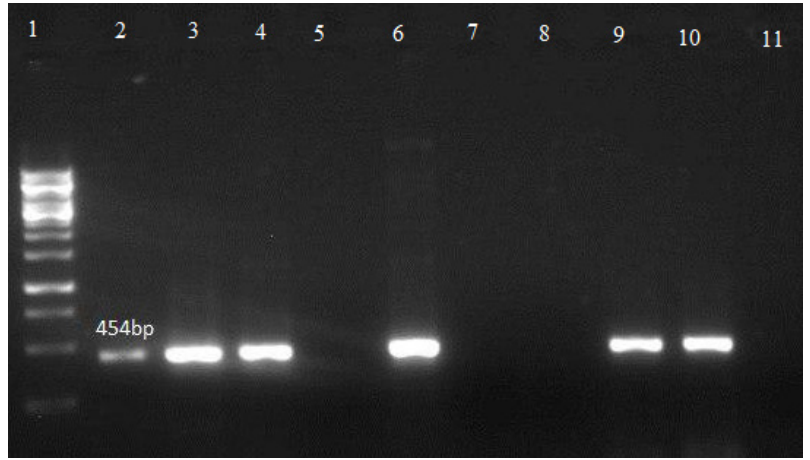

Fig. 4. Agarose gel electrophoresis showing band patterns showing sld gene Lane 1: DNA marker (1000 bp Ladder) Lane 10: positive control

Lane 11: negative control

Lanes 2-4, 6 and 9: DNA samples positive for sld gene

Lanes 5, 7 and 8: DNA samples negative for sld gene $(\mathrm{p}=0.917)$, and pho $(\mathrm{p}=0.283)$ genes in $\mathrm{G}$. vaginalis associated with non-BV and BV cases was not statistically significant (Table 2).

\section{Discussion}

Vaginal econiche harbors a complex population of bacteria which may vary in different medical conditions. A shift from the predominant Lactobacillus spp. in healthy vaginal environment may lead to $\mathrm{BV}$. The complete mechanism underlying BV pathogenicity is not clear due to the lack of an animal model. One microorganism which has been shown to be strongly associated with BV is G. vaginalis. However, despite the fact that G. vaginalis

\begin{tabular}{|c|c|c|c|c|c|c|}
\hline & & & $\begin{array}{c}\text { non- } B V \text { associated } \\
\text { G. vaginalis }\end{array}$ & $\begin{array}{l}\text { BV associated } \\
\text { G. vaginalis }\end{array}$ & Total & $\mathrm{p}$ \\
\hline \multirow[t]{6}{*}{ vly } & Pos & Count & 20 & 10 & 30 & 0.324 \\
\hline & & $\%$ within disease & $47.6 \%$ & $35.7 \%$ & $42.9 \%$ & \\
\hline & Neg & Count & 22 & 18 & 40 & \\
\hline & & $\%$ within disease & $52.4 \%$ & $64.3 \%$ & $57.1 \%$ & \\
\hline & Total & Count & 42 & 28 & 70 & \\
\hline & & $\%$ within disease & $100.0 \%$ & $100.0 \%$ & $100.0 \%$ & \\
\hline \multirow[t]{6}{*}{ sld } & Pos & Count & 28 & 19 & 47 & 0.917 \\
\hline & & $\%$ within disease & $66.7 \%$ & $67.9 \%$ & $67.1 \%$ & \\
\hline & Neg & Count & 14 & 9 & 23 & \\
\hline & & $\%$ within disease & $33.3 \%$ & $32.1 \%$ & $32.9 \%$ & \\
\hline & Total & Count & 42 & 28 & 70 & \\
\hline & & $\%$ within disease & $100.0 \%$ & $100.0 \%$ & $100.0 \%$ & \\
\hline \multirow[t]{6}{*}{ pho } & Pos & Count & 5 & 6 & 11 & 0.283 \\
\hline & & $\%$ within disease & $11.9 \%$ & $21.4 \%$ & $15.7 \%$ & \\
\hline & Neg & Count & 37 & 22 & 59 & \\
\hline & & $\%$ within disease & $88.1 \%$ & $78.6 \%$ & $84.3 \%$ & \\
\hline & Total & Count & 42 & 28 & 70 & \\
\hline & & $\%$ within disease & $100.0 \%$ & $100.0 \%$ & $100.0 \%$ & \\
\hline
\end{tabular}


is the most prevalent and virulent species found in BV, the normal vaginal microbiota can also consist of this microorganism.

There was an assumption that there may be a diversity within this species, as $G$. vaginalis can also colonize the lower genital tract of healthy women. There are many studies regarding the pathogenesis of $G$. vaginalis which led to the classification of this microorganism into 8 biotypes based on the phenotypic visualization of enzymes including galactosidase, lipase, and hippurate hydrolase (27). Amplified rDNA restriction analysis (ARDRA) was used for genotypic classification in which 3 genotypes have yet been recognized (28). It has been proposed that some of the known $G$. vaginalis genotypes are, in fact, distinct species. Whole-genome sequence analysis and functional microbiology genomics corroborate this idea (29).

In the present study, standard methods, including Nugent score and Amsel's criteria were used for BV diagnosis. Nugent scoring system measures the quantity of bacterial morphotypes in Gram-stained vaginal smears. This scoring system is based on the absence or presence of Lactobacillus spp., Mobiluncus spp. and G. vaginalis and their total quantity (average per microscopic field). In the present study, the prevalence of BV based on these diagnostic methods was shown to be $27.4 \%$.

Due to the polymicrobial nature of the vaginal ecosystem and the competition for growth in culture media, complex nutritional requirement and slow growth of the microorganism, isolation of $G$. vaginalis is challenging. Thus, culture-based identification is laborious and time-consuming. In the present study, in addition to bacterial culture, PCR-based techniques using the $16 \mathrm{~S}$ rRNA sequence was used in direct vaginal fluid samples for detection of $G$. vaginalis. The aforementioned facts caused the difference in the prevalence of the microorganism in direct vaginal samples and culture media. It is evident that detection in the direct vaginal sample by PCR was more efficient. Gardnerella vaginalis bears various virulence factors including sialidase, phospholipase $\mathrm{C}$, and vaginolysin. It also has been suggested that this bacterium has other pathogenicity determinants, such as surface hydrophobicity, adherence properties, biofilm formation and prolidase activity (11, 29) which were not looked at in this study.

In this investigation, the occurrence of vly gene was shown to be $35.7 \%$ among $28 \mathrm{BV}$ patients and $47.6 \%$ among the 42 healthy women. This did not show any significant correlation $(\mathrm{p}>0.05)$. This prevalence was lower compared to the studies conducted in Portugal and Brazil. They did not find any significant association between the prevalence of this gene and $\mathrm{BV}$ infection as well $(29,30)$.

The prevalence of sld gene, was $67.8 \%$ and $66.6 \%$ in $\mathrm{BV}$ and non-BV associated $G$. vaginalis, respectively. There too was no significant correlation among the two groups, which was in accordance with other studies (29). Nada Khairi Younus et al. (31) studied 47 G. vaginalis isolated from 207 patients with presumed BV and found biotypes 1 and 7 as the most common isolates. Amplified
rDNA restriction analysis showed that all strains with genotypes $1(n=12)$ and $3(n=13)$ had sld genes. The prevalence of vly gene in these genotypes was $91.6 \%$ and $84.6 \%$, respectively. Milda Pleckaityte et al. (32) found vly gene in all of their $17 \mathrm{G}$. vaginalis isolates; however, they could not find a correlation between genotypes and vly positiveness.

Phospholipase $\mathrm{C}$ gene was the other studied virulence gene with its frequency in BV and non-BV associated $G$. vaginalis detected to be $21.4 \%$ and $11.9 \%$, respectively. This showed a lack of significant relationship with the disease $(p>0.05)$. There was no prior study regarding the prevalence of this gene, so it is not possible to compare the results. However, some studies showed phospholipase $\mathrm{C}$ production by different methods including a study which showed phospholipase C production in $22 \%$ of $G$. vaginalis associated with $\mathrm{BV}$ and at $27 \%$ in normal flora on skim milk agar (33). Also, in a study conducted by J. Udayalaxmi et al. (34), out of the 32 isolates associated with BV, $28(87.5 \%)$ produced phospholipase $\mathrm{C}$ by use of the synthetic substrate p-nitrophenylphosphorylcholine. In overall, detection of $G$. vaginalis depends on the use of various tools and techniques. There have not been sufficient studies on the prevalence and pathogenicity of this microorganism in Iran.

\section{Conclusion}

The results of this study show a high prevalence of $G$. vaginalis in patients with $\mathrm{BV}$ and relatively high prevalence in healthy individuals. The presence of virulence genes suggests the pathogenic potentials of this microorganism. Also, molecular techniques for the detection of $G$. vaginalis was found to be more efficient as the culture approach is time-consuming and requires a lot of efforts. A shortcoming of this study was the lack of biotyping and ARDRA genotyping for the establishment of a correlation between the occurrence of the studied virulence genes and different biotypes and genotypes of $G$. vaginalis. Further studies regarding the expression of these virulence genes may be useful to evaluate the conditions leading to the production of these virulence factors and their prevalence in BV patients and healthy women. Also, due to lack of reports about the prevalence rate of resistance genes and typing of this microorganism in Iran, future studies on these subjects are recommended which can provide useful data for better treatment of BV.

\section{Acknowledgments}

This study was financially supported by a research grant (No.96-01-30-29898) for an M.Sc thesis at Iran University of Medical Sciences (Tehran, Iran), for which we are very grateful.

\section{Conflict of Interests}

The authors declare that they have no competing interests.

\section{References}

1. Hay P. Bacterial vaginosis. F1000Research [Internet]. 2017;6:1761. Available from: https://f1000research.com/articles/6-1761/v1. 
2. Feng S, ZhengAi X. Research progress of mechanisms of bacterial vaginosis on pregnancy outcomes. Chinese $\mathrm{J}$ Microecol. 2013;25:365-7.

3. Lewis FMT, Bernstein KT, Aral SO. Vaginal microbiome and its relationship to behavior, sexual health, and sexually transmitted diseases. Obstet Gynecol. 2017;129(4):643-54.

4. Patterson JL, Stull-Lane A, Girerd PH, Jefferson KK. Analysis of adherence, biofilm formation and cytotoxicity suggests a greater virulence potential of Gardnerella vaginalis relative to other bacterialvaginosis-associated anaerobes. Microbiology. 2010;156(2):392-9.

5. Mendling W. Normal and abnormal vaginal microbiota. LaboratoriumsMedizin. 2016;40(4):239-46.

6. Abdelmaksoud AA, Girerd PH, Garcia EM, Brooks JP, Leftwich LM, Sheth NU, et al. Association between statin use, the vaginal microbiome, and Gardnerella vaginalis vaginolysin-mediated cytotoxicity. PLoS One [Internet]. 2017;12(8):1-17.

7. Margolis E, Fredricks DN. Bacterial Vaginosis-Associated Bacteria. In: Molecular Medical Microbiology: Second Edition. 2014. p. 148796.

8. Menard JP, Mazouni C, Salem-Cherif I, Fenollar F, Raoult D, Boubli $\mathrm{L}$, et al. High vaginal concentrations of atopobium vaginae and gardnerella vaginalis in women undergoing preterm labor. Obstet Gynecol. 2010;115(1):134-40.

9. Catlin BW. Gardnerella vaginalis: Characteristics, clinical considerations, and controversies. Clin Microbiol Rev. 1992;5:21337.

10. Srinivasan S, Hoffman NG, Morgan MT, Matsen FA, Fiedler TL, Hall RW, et al. Bacterial communities in women with bacterial vaginosis: High resolution phylogenetic analyses reveal relationships of microbiota to clinical criteria. PLoS One. 2012;7(6).

11. Harwich MD, Alves JM, Buck GA, Strauss JF, Patterson JL, Oki $\mathrm{AT}$, et al. Drawing the line between commensal and pathogenic Gardnerella vaginalis through genome analysis and virulence studies. BMC Genomics. 2010;11(375):1-12.

12. Yoon HJ, Chun J, Kim JH, Kang SS, Na DJ. Gardnerella vaginalis septicaemia with pyelonephritis, infective endocarditis and septic emboli in the kidney and brain of an adult male. Int J STD AIDS. 2010;21(9):653-7.

13. Neri P, Salvolini S, Giovannini A, Mariotti C. Retinal vasculitis associated with asymptomatic gardnerella vaginalis infection: A new clinical entity. Ocul Immunol Inflamm. 2009;17(1):36-40.

14. Sivadon-Tardy V, Roux AL, Piriou P, Herrmann JL, Gaillard JL, Rottman M. Gardnerella vaginalis acute hip arthritis in a renal transplant recipient. J Clin Microbiol. 2009;47(1):264-5.

15. Graham S, Howes C, Dunsmuir R, Sandoe J. Vertebral osteomyelitis and discitis due to Gardnerella vaginalis. J Med Microbiol. 2009;58(10):1382-4.

16. Lagacé-Wiens PRS, Ng B, Reimer A, Burdz T, Wiebe D, Bernard $\mathrm{K}$. Gardnerella vaginalis bacteremia in a previously healthy man: Case report and characterization of the isolate. J Clin Microbiol. 2008;46(2):804-6.

17. Gelber SE, Aguilar JL, Lewis KLT, Ratner AJ. Functional and phylogenetic characterization of vaginolysin, the human-specific cytolysin from Gardnerella vaginalis. J Bacteriol. 2008;190(11):3896-903.

18. Hughes TR, Ross KS, Cowan GJM, Sivasankar B, Harris CL, Mitchell TJ, et al. Identification of the high affinity binding site in the Streptococcus intermedius toxin intermedilysin for its membrane receptor, the human complement regulator CD59. Mol Immunol. 2009;46(7):1561-7.

19. Ohkuni H, Nagamune H, Ozaki N, Tabata A, Todome Y, Watanabe $\mathrm{Y}$, et al. Characterization of recombinant Streptococcus mitis-derived human platelet aggregation factor. APMIS. 2012;120(1):56-71.

20. Randis TM, Kulkarni R, Aguilar JL, Ratner AJ. Antibody-based detection and inhibition of vaginolysin, the Gardnerella vaginalis cytolysin. PLoS One. 2009;4(4):1-5.

21. Zilnyte M, Venclovas Č, Zvirbliene A, Pleckaityte M. The cytolytic activity of vaginolysin strictly depends on cholesterol and is potentiated by human CD59. Toxins (Basel). 2015;7(1):110-28.

22. Zilnyte M, Venclovas eslovas, Zvirbliene A, Pleckaityte M. The cytolytic activity of vaginolysin strictly depends on cholesterol and is potentiated by human CD59. Toxins (Basel). 2015;7(1):110-28.

23. Cauci S, Culhane JF, Di Santolo M, McCollum K. Among pregnant women with bacterial vaginosis, the hydrolytic enzymes sialidase and prolidase are positively associated with interleukin- $1 \beta$. Am J Obstet
Gynecol. 2008;198(1)

24. Udayalaxmi J, Bhat GK, Kotigadde S. Biotypes and virulence factors of Gardnerella vaginalis isolated from cases of bacterial vaginosis. Indian J Med Microbiol. 2011;29(2):165-8.

25. Nugent Rp, Krohn MA, Hillier SL. Reliability of Diagnosing Bacterial Vaginosis Is Improved by Standardized Method of Gram Stain Interpretation a. Am Soc Microbiol. 1991;29(2):297-301.

26. Amsel R, Totten PA, Spiegel CA, Chen KCS, Eschenbach D, Holmes KK. Nonspecific vaginitis. Diagnostic criteria and microbial and epidemiologic associations. Am J Med. 1983;74(1):14-22.

27. Piot P, van Dyck E, Peeters M, Hale J, Totten PA, Holmes KK. Biotypes of Gardnerella vaginalis. J Clin Microbiol. 1984;20(4):677-

28. Ingianni A, Petruzzelli S, Morandotti G, Pompei R. Genotypic differentiation of Gardnerella vaginalis by amplified ribosomal DNA restriction analysis (ARDRA). FEMS Immunol Med Microbiol. 1997;18(1):61-6.

29. Castro J, Alves P, Sousa C, Cereija T, França Â. Using an in-vitro biofilm model to assess the virulence potential of Bacterial Vaginosis or non- Bacterial Vaginosis Gardnerella vaginalis isolates. Nat Publ Gr. 2015;(June):1-10.

30. Maria D, Souza K De, Diniz CG, Silveira D, Filho C, Maria L. Antimicrobial susceptibility and vaginolysin in Gardnerella vaginalis from healthy and bacterial vaginosis diagnosed women. J Infect Dev Ctries. 2016;10(09):913-9.

31. Younus NK, Gopinath R, Jegasothy R, Nordin SA, van Belkum A, Mary N, et al. An update on Gardneralla vaginalis associated bacterial vaginosis in Malaysia. Asian Pac J Trop Biomed. 2017:7(9):831-5.

32. Pleckaityte M, Janulaitiene M, Lasickiene R, Zvirbliene A. Genetic and biochemical diversities of Gardnerella vaginalis strains isolated from women with bacterial vaginosis. Immunol Med Microbiol 2012;65(1):69-77

33. Chlamydia DOF, Infection T, Young A, Of P, Vaginalis G, With A, et al. P3 . 11 Biotypes, biofilm and phospholipase c production of gardnerella vaginalis associated with normal flora and bacterial vaginosis. Sex Transm Infect. 2017;93(Suppl 2):10-1.

34. Udayalaxmi J, Bhat GK, Kotigadde S. Biotypes and virulence factors of Gardnerella vaginalis isolated from cases of bacterial vaginosis. Indian J Med Microbiol. 2011;29:165-9. 